\title{
AN OPTIMIZATION ALGORITHM BASED ON GRID-GRAPHS FOR MINIMIZING INTERCONNECT DELAY IN VLSI LAYOUT DESIGN
}

\author{
Mohamed Khalil-Hani ${ }^{1}$, Nasir Shaikh-Husin ${ }^{2}$ \\ VeCAD Research Laboratory \\ Faculty of Electrical Engineering \\ Universiti Teknologi Malaysia (UTM) \\ 81310 UTM Skudai, Johor, Malaysia. \\ khalil@fke.utm.my ${ }^{1}$,nasirsh@utm.my ${ }^{2}$
}

\begin{abstract}
In this paper, we describe a routing optimization algorithm based on grid-graphs for application in a deep-submicron VLSI layout design. The proposed algorithm, named S-RABILA (for

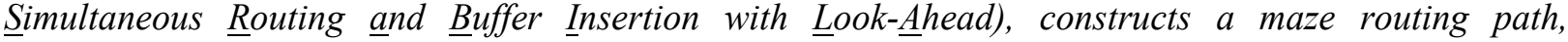
simultaneously with buffer insertion and wire sizing, taking into account wire and buffer obstacles, such that the interconnect delay from source to sink is minimized. In current nanometer VLSI layout design, the interconnect delay has become the dominant factor affecting system performance. Research has shown that routing algorithms, which include simultaneous buffer insertion and wire-sizing, have been proven to be very effective in solving the timing optimization problem in VLSI interconnect design. A key contribution of this work is a novel look-ahead scheme applied to speed up the runtime of the algorithm, and aids in finding the exact solution. Hence, the algorithm is accurate, fast, scalable with problem size, and can handle large routing graphs. Experimental results show the effectiveness of the look-ahead scheme and indicate that S-RABILA provides significant performance improvements over similar existing VLSI routing algorithms.
\end{abstract}

Keywords: Interconnect optimization, VLSI routing, Buffer insertion, Elmore delay.

\section{I.0 INTRODUCTION}

In deep submicron fabrication technology, transistors can now switch much faster, but wire resistances are now larger, and delay due to wires can exceed gate delay. Consequently, the interconnect delay is the dominant factor in the construction of wire routing in very large scale integrated (VLSI) circuits, which today, has feature dimensions in the nanometer range. Today, the state-of-the-art circuit design involves as much the engineering of the wires as the design of transistors. Hence, a successful VLSI design today depends heavily on a successful interconnect design.

An effective approach for reducing the interconnect delay is buffer insertion [1]. In this method, a wire is divided into segments with a buffer inserted between the segments [2]. Traditionally, buffer insertion is a post-layout optimization technique, implying that the routing paths are first found, and then buffers are inserted in these paths. However, today's VLSI designs typically apply some form of design reuse utilizing pre-designed cells, or macro blocks. Clearly, buffers cannot be inserted into areas in the VLSI layout occupied by these macro blocks (here on, referred to as "buffer obstacle" areas). However, it is possible to route a wire over these buffer obstacles (e.g. through a higher level interconnect). Nevertheless, a wire routed for long stretches over the buffer obstacle areas can result in a huge wire delay. Hence, the buffer insertions must take into account these buffer obstacle areas. It is clear that the two-stage "routing then insert buffer" approach cannot give an optimal solution; that is, with the macro blocks, a shortest path cannot guarantee minimum delay. Furthermore, since a wire adds loading to a buffer, proper wire sizing can result in minimum delay. Proper wire sizing can reduce the interconnect delay by a further $10 \%$ [3]. For nets that have macro blocks where wires can pass through but not buffers, we can find the optimal interconnect path if routing, buffer insertion, and wire sizing are considered 
simultaneously. The problem at hand is essentially an interconnect optimization problem, and is conceptually summarized as follows. Given a source and sink node of a net in a VLSI layout, we wish to find a buffered routing path that connects these two nodes such that the end-to-end delay is a minimum.

This paper proposes an efficient algorithm to solve the above interconnect optimization problem. The key goal of the algorithm is to construct a maze routing path, simultaneously with buffer insertion and wire sizing in the presence of wire and buffer obstacles, such that the Elmore delay from source to sink is minimized. The problem is formulated as a shortest-path problem in a weighted graph. The proposed

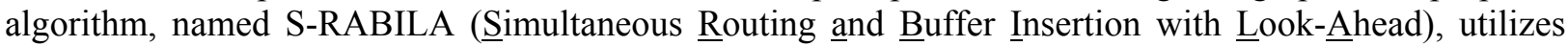
three key concepts in order to find a solution efficiently. They are $k$-shortest path search, dominant path, and a novel look-ahead feature. This look-ahead scheme, which significantly speeds up execution time of the algorithm, is a key contribution of this work. The result is that the proposed algorithm is accurate, fast, scalable with problem size, and can handle large routing graphs. The paper is organized as follows. In Section 2, previous work that are related to this work are presented, followed by, in Section 3, a discussion on the buffered path search problem, including Elmore delay formulations as applied in this work. Section 4 describes the proposed algorithm, S-RABILA. The experimental result of the performance tests conducted on S-RABILA is provided in Section 5. Finally, we present the conclusion in Section 6.

\subsection{PREVIOUS RELATED WORK}

Many algorithms have been proposed to solve the interconnect optimization problem. van Ginneken pioneered the optimum buffering in a routing tree structure [1]. This algorithm, which applies dynamic programming, can only insert buffers after the routing tree is known. An existing algorithm called Fast Buffer Insertion (FBI), proposed by Li and Shi [4], inserts buffers optimally for a given routing tree. This algorithm can accommodate multiple buffers, although it cannot handle multiple wires. However, these algorithms are post-routing buffer insertion techniques. Lillis, Cheng, and Lin have enhanced the van Ginneken algorithm to obtain even better delay optimality by solving the routing problem with simultaneous buffer insertion and wire sizing [5].

More recently, much improved algorithms for simultaneous routing and buffer insertion have been proposed, that are more suitable for today's VLSI technology. Zhou et al. proposed such an algorithm, in which the routing path is obtained with simultaneous buffer insertion, and in addition, took into account wire and buffer obstacles [6]. Since the search technique applied is based on van Ginneken dynamic programming approach, we shall refer to Zhao's algorithm as the DP algorithm in this paper. This algorithm was further enhanced to include wire sizing by Lai and Wong [3]. They call their algorithm SP, for Shortest Path algorithm. SP is a graph-based algorithm, first converts the input maze graph into a bigger graph, which is called buffer planning (BP) graph. Dijkstra's algorithm is then applied on the BP graph to find the optimal delay path, which is determined to be the shortest path between the source and sink vertices in the BP graph. These algorithms mentioned above utilized Elmore delay models. In [7], Huang et al. extended the work by Lai and Wong [3] to include wire inductance using transmission line model for the delay computation. However, only fixed wire sizes are considered, and the solution is restricted to small problems. It should be noted here, that the DP and SP algorithms mentioned above are used in benchmarking the performance of S-RABILA algorithm proposed in this paper.

A serious limitation of these existing algorithms is that their execution times are not adequately fast for application in today's deep-submicron VLSI layout design, particularly when applied to large problems. They do not use look-ahead schemes, or the like, which can speed up the algorithm runtimes, and furthermore, they cannot provide exact solutions. In this aspect, Van Mieghem and Kuipers have proposed an algorithm called SAMCRA that uses a novel look-ahead concept in finding an exact solution in multi-weighted graph problems applied in Quality-of-Service (QoS) routing [8]. A similar idea is applied in $\mathrm{A}^{*}$ graph search algorithm for single weight graphs [11]. This look-ahead concept is adapted in this work for application in VLSI routing problems. 


\subsection{BUFFERED PATH SEARCH PROBLEM}

The maze routing with simultaneous buffer insertion and wire sizing problem in VLSI layout design is essentially a buffered routing path search problem. In this work, it is formulated as a shortest-path problem in a weighted graph, and is specified as follows. Given a routing grid graph $G=(V, E)$ corresponding to a VLSI layout, a buffer library $B$, a buffer function $p$ with $p(v)=1$ indicating buffer insertion is allowed at vertex $v$, a wire library $W$, two vertices $s$, sink $\in V$, find a buffered path $P=\left(v_{1}, v_{2}\right.$, $\left.\ldots, v_{n}\right)$, with $v_{1}=s, v_{n}=\sin k, b\left(v_{i}\right) \in B \cup\{-1\}$ where $b\left(v_{i}\right)=-1$ indicates that no buffer is inserted at $v_{i}$, and $w\left(v_{j}, v_{j+1}\right) \in W$, such that the interconnect delay for path $P$ is minimized. $V=\{s\} \cup\{\operatorname{sink}\} \cup V_{n}$ and $E$ is the set of edges. Vertex $s$ is the source vertex, sink is the sink vertex, and $V_{n}$ is the set of internal vertices. A vertex $v \in V_{n}$ may belong to the set of buffer obstacle vertices (vertices in areas where buffers are not allowed), denoted $V_{O B}$, or to the set of wire obstacle vertices (vertices in areas where wires are not allowed), denoted as $V_{O W}$. A buffer library $B$ contains different types of buffer and a wire library $W$ contains wires with different widths. A buffer in library $B$ is denoted $B_{i}$ and a wire in wire library $W$ is denoted $W_{i}$. For each edge $e=u \rightarrow v$, signal travels from $u$ to $v$, where $u$ is the upstream vertex while $v$ is the downstream vertex and $u, v \notin V_{O W}$.

Consider a sample problem shown in Fig. 1, where the grid graph, buffer obstacles (grey areas) and wire obstacles (dark areas) are given. The model parameters used are as follows: source resistance is $140 \Omega$, load capacitance at the sink is $0.002 \mathrm{pF}$, and resistance and capacitance of a wire segment are $58 \Omega$ and $0.042 \mathrm{pF}$ respectively. We assume there is only one type of buffer with input capacitance, output resistance and intrinsic delay of $0.002 \mathrm{pF}, 140 \Omega$ and $40 \mathrm{ps}$ respectively. One approach of the path search is simply to find the shortest distance between the source and sink points without going into obstacle areas. Once the path is determined, buffers are placed along this path so that the delay is minimized. The result on our sample problem is depicted in Fig. 1(a), where the total path delay is found to be 680.6 ps. Another method is to ignore only wire obstacles (i.e. buffer obstacles are taken into account) and find the shortest path between the end points. Once this path is found, buffers are placed at the allowable positions. The resulting path is shown in Fig. 1(b), and the total path delay is found to be $621.8 \mathrm{ps}$, which is an improvement over the previous case. Both these cases apply post-routing buffer insertion techniques.

The best result is obtained by applying both path search and buffer insertion simultaneously. The optimum solution where buffer restrictions are considered when searching for the best path is shown in Fig. 1(c). The total path delay in this case is found to be $521.7 \mathrm{ps}$, which is a significant improvement. This clearly indicates that simultaneous routing and buffer insertion is the approach to take in order to find the optimal delay routing. The problem however becomes more challenging if we have several buffer types to choose from. Each buffer differs in terms of input capacitance, output resistance and intrinsic delay. The problem complexity further increases when we consider several wire sizes (i.e. different resistance and capacitance characteristics) to connect any buffer-to-buffer section. In these latter cases, where buffer types and wire sizes are considered, the runtime of existing algorithms are unacceptable, especially in large problems. 


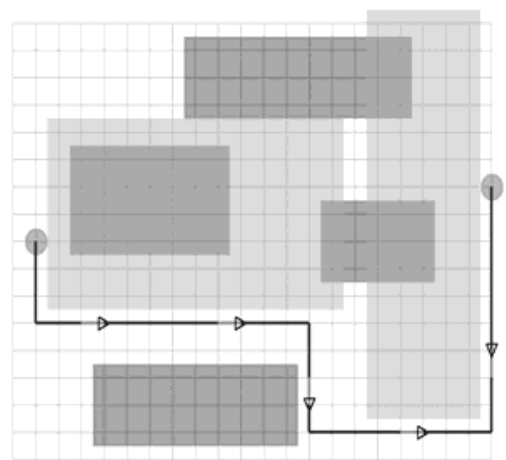

(a)

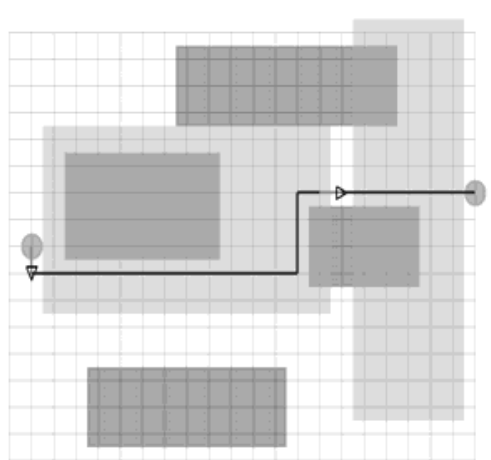

(b)

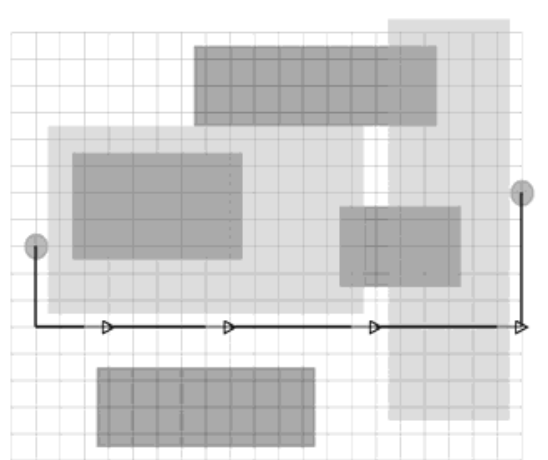

(c)

Fig. 1. A sample maze routing problem (the dark areas are wire obstacles while the grey areas are buffer obstacles.) (a) Routing path avoids all obstacles. (b) Routing path with buffer obstacles considered. (c) Optimal routing. 


\subsection{Interconnect Delay Model Formulations}

Recalling the problem at hand, we wish to find a buffered path from a source to a sink node such that the interconnect delay is minimized. For the target technology (i.e. deep sub-micron VLSI), where wire resistance can no longer be ignored, an interconnect is typically modeled as an RC distributed network. A wire segment in the network is represented by a $\pi$-model RC circuit as shown in Fig. 2(a), and a buffer is modeled by the circuit shown in Fig. 2(b). The labels $r_{w}$ and $c_{w}$ are resistance and capacitance of the wire segment, and $c_{b}, r_{b}$, and $d_{b}$ are input capacitance, output resistance, and intrinsic delay of the buffer.

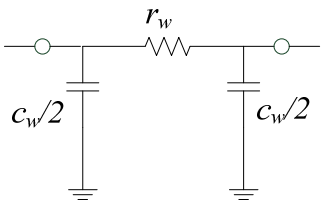

(a)

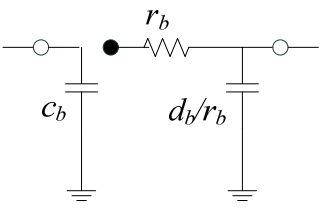

(b)

Fig. 2. (a) Wire segment model. (b) Buffer model.

In this work, Elmore delay is used to model the interconnects. Elmore delay has excellent correlation to the actual delay, and in fact, it is the upper bound for the actual delay [9]. This work applies an important property of Elmore delay that allows its computation to be performed incrementally and iteratively. The technique is based on the equations formulated by van Ginneken [1], which we now summarized. At the outset, note that we will have different Elmore delay equations for the case when the path expansion begins from the source node and progresses to the sink, and for the case when the path expansion begins from the sink and ending at the source.

In path expansion scheme beginning at the source node, each node in the wire is labeled with a resistance-delay pair $(r, t)$, where $r$ and $t$ are the resistance and delay accumulated up to that segment, respectively [3], [6]. Given a resistance-delay pair value $(r, t)$ at the output of a wire segment, the resistance-delay pair value at the output of the subsequent downstream segment $\left(r^{\prime}, t^{\prime}\right)$ can be computed as follows. If the downstream segment is a wire, then $\left(r^{\prime}, t^{\prime}\right)$ is

$$
r^{\prime}=r_{w}+r, \quad \text { and } \quad t^{\prime}=\left(r+r_{w} / 2\right) c_{w}+t .
$$

If the downstream segment is of a wire terminated with a buffer, then $\left(r^{\prime}, t^{\prime}\right)$ is

$$
r^{\prime}=r_{b}, \quad \text { and } \quad t^{\prime}=r\left(c_{w}+c_{b}\right)+r_{w}\left(c_{w} / 2+c_{b}\right)+d_{b}+t .
$$

In contrast, in the path expansion scheme beginning at the sink node, instead of a resistance-delay pair, each node $v$ is labeled with a capacitance-delay pair, $(c, t)$, where $c$ represents the total ground capacitance $C_{T}(v)$ rooted at node $v$. With an initial $(c, t)$ given, a new capacitance-delay pair $\left(c^{\prime}, t^{\prime}\right)$ for the preceding segment can be determined, as follows. If the preceding upstream segment is a wire only, then $\left(c^{\prime}, t^{\prime}\right)$ is given by

$$
c^{\prime}=c_{w}+c, \quad \text { and } \quad t^{\prime}=r_{w}\left(c_{w} / 2+c\right)+t .
$$

If the preceding segment is a wire terminated with a buffer, then new capacitance-delay pair becomes

$$
c^{\prime}=c_{w}+c_{b}, \quad \text { and } \quad t^{\prime}=r_{w}\left(c_{w} / 2+c_{b}\right)+d_{b}+r_{b} c+t .
$$

This suggests that we can perform the computations for both path traversals concurrently. We maintain the $(r, t)$ pairs at the nodes for the path traversal originating from the source, while $(c, t)$ pairs are maintained at the nodes for the path traversal originating from the sink. The end-to-end delay (denoted by 
EndDelay) is obtained, when both $(r, t)$ and $(c, t)$ pairs are available at a particular node. Hence, at node $M$, where both $(r, t)$ and $(c, t)$ pairs are already determined,

$$
\text { EndDelay }=t_{M}+t_{m}+r_{M} c_{M}
$$

where $\left(r_{M}, t_{M}\right)$ is the resistance-delay pair, and $\left(c_{M}, t_{m}\right)$ is the capacitance-delay pair values at node $M$.

\section{2 $\quad k$-Shortest Path Search}

The routing problem at hand now becomes a multi-weighted single constraint graph problem. Each routing path is weighted with either resistance-delay pair or capacitance-delay pair, depending on the direction of path expansion. Dijkstra's algorithm cannot be applied to graphs with multi-weight edges, as the algorithm requires the property that the shortest path must consist of shortest sub-paths [10]. In multiweight graphs the shortest path to any vertex is not unique anymore. Consider the example given in Fig. 3 , which shows a two-weighted graph. There are two shortest paths to reach vertex $d$ from $a$. The cost for path $a \rightarrow b \rightarrow d$ is $(5,6)$ while the cost for path $a \rightarrow c \rightarrow d$ is $(3,7)$. The first path has a lower second weight while the other path has a lower first weight. We should not discard either path in our search for the final solution.

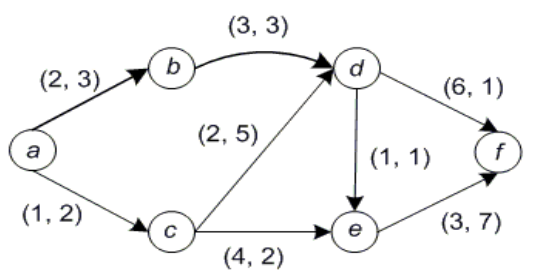

Fig. 3. A multi-weight graph.

Therefore, when dealing with a multi-weight graph, it is necessary to store multiple sub-paths for any vertex. There are multiple valid shortest paths in reaching a vertex; each path has at least one lower weight component compared to other paths stored for that vertex. This gives rise to so-called " $k$-shortest path" approach. In the problem here, since each intermediate node is characterized by two weights for our Elmore delay calculation, we have to store the shortest delay, second shortest delay, third shortest delay, etc., up to $k$-shortest delay path for each intermediate node. If $k$ is not restricted, a $k$-shortest path algorithm returns all possible paths from source to the destination. An algorithm may restrict the limit for $k$, for example in order to reduce memory requirements and runtime. However, this algorithm cannot guarantee end-to-end shortest delay path anymore [8]. This dilemma is compounded by the fact that the parameter $k$ is different for each intermediate node. Fixing $k$ to a large value may result in unnecessary large memory allocation for most nodes that do not require a large $k$. To guarantee optimal end-to-end delay and efficient memory allocation, S-RABILA determines the parameter $k$ adaptively for each intermediate node. The fact that S-RABILA does not restrict the parameter $k$, alluding to computation of all feasible paths between source and destination, gives an impression that the algorithm may exhibit NPcomplete traits. However, the number of feasible paths can be reduced significantly by applying the "path-dominance" property.

As mentioned above, in the construction of the routing path, a vertex $u$ may have several path candidates (or sub-paths), which correspond to different combinations of wire and buffer sizes for the paths that reach vertex $u$. Each candidate is weighted with a resistance-delay pair $(r, t)$, where $r$ is the accumulated resistance at vertex $u$ (from the source to $u$ ) and $t$ is the delay up to vertex $u$. A new candidate is added to the list of existing candidates if it is not dominated. Between any two candidates $\alpha_{1}=\left(r_{1}, t_{1}\right)$ and $\alpha_{2}=\left(r_{2}\right.$, $\left.t_{2}\right), \alpha_{1}$ is said to "dominate" $\alpha_{2}$ if $t_{1} \leq t_{2}$ and $r_{1} \leq r_{2}$. The "dominated" candidate $\alpha_{2}$ is not added or, if already in the list, it is removed. This check for path-dominance property between the candidates stored at a vertex is essentially a state space reduction technique. It contributes significantly to an efficient path search, and is applied in S-RABILA. 


\subsection{S-RABILA ALGORITHM}

The proposed algorithm, S-RABILA solves the simultaneous maze routing and buffer insertion problem in a VLSI layout design. The goal is to find a buffered path (with wiresizing) between the source and a sink such that the interconnect delay of the routed path is minimized. As in other maze routing, the entire routing area is represented as a 2-dimensional (2D) grid graph, where areas of buffer and wire obstacles are known, and the source and destination vertices are specified. The top-level behavior of S-RABILA algorithm is shown in Fig. 4, and its MAIN() procedure in pseudo-code is provided in Fig. 5.

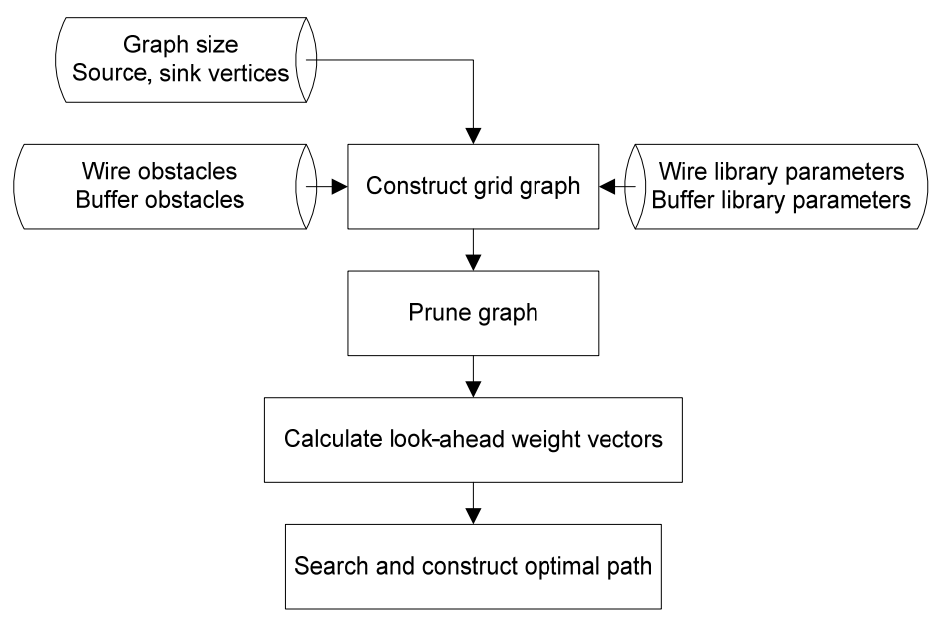

Fig. 4. Top-level description of S-RABILA algorithm.

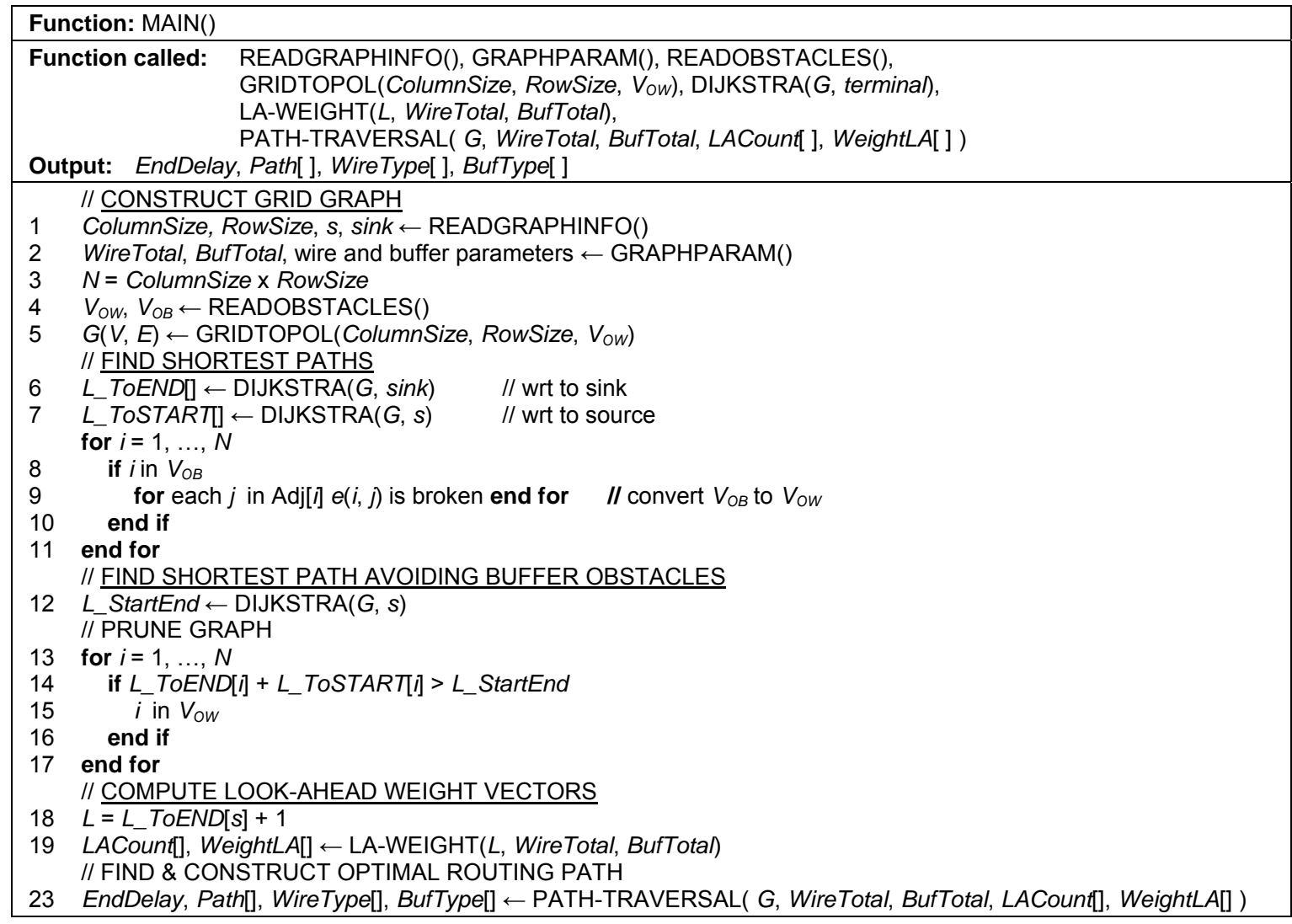

Fig. 5. MAIN( ) routine of S-RABILA. 
The algorithm is made up of four core stages. Stage 1 involves the extraction of the graph parameters and formation of the problem grid graph. Based on these parameters, the grid graph $G(N, E)$ modeling the problem, with $N$ vertices and $E$ edges, is formed through the GRIDTOPOL() subroutine. The second stage performs graph pruning. Stage three involves the computation of the look-ahead weight vectors. The look-ahead weight vectors are obtained by executing the function LA-WEIGHT(). Finally, in stage 4 of the algorithm, the required optimal routing path is searched, traced, and constructed, through the execution of the PATH-TRAVERSAL() meta-function. The path search using the PATHTRAVERSAL() function is illustrated via flowchart diagram in Fig. 6.

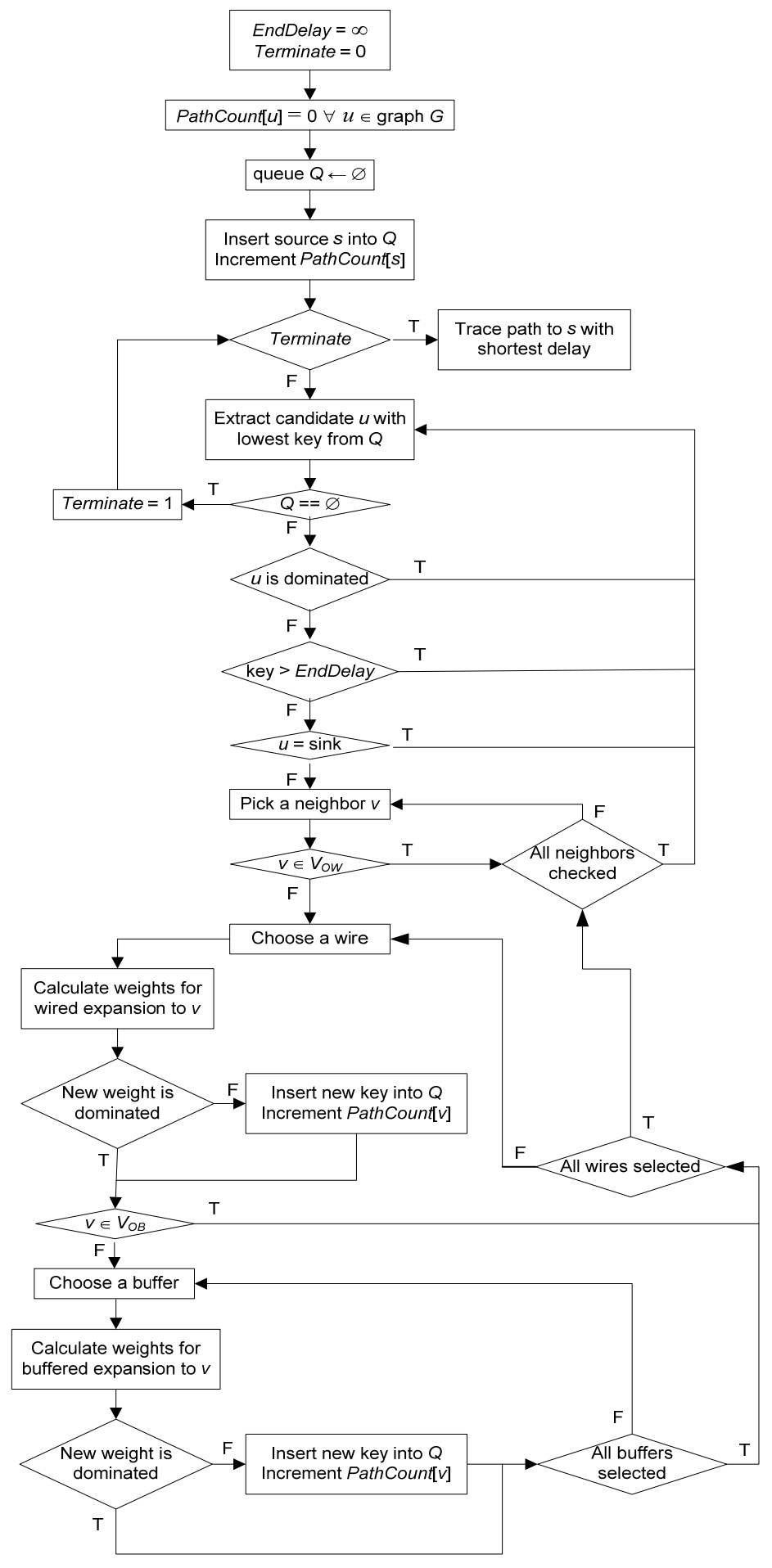

Fig. 6. Flowchart for PATH-TRAVERSAL meta-function. 


\subsection{Graph Pruning}

The effort in constructing a routing path depends on the search space of the graph. We can reduce the effort by pruning the graph, whereby redundant vertices are removed from the graph. In S-RABILA, a vertex is considered redundant in the search space if $L_{-} T o E N D[u]+L_{-}$ToSTART $[u]>L \_$StartEnd, where $L_{-} T o E N D[u]$ is the shortest path length from vertex $u$ to the sink vertex $E N D$ while L_ToSTART $[u]$ is the shortest path length from vertex $u$ to the source vertex START. These paths can pass through areas where buffer is not allowed (i.e. $V_{O B}$ ). Now we define a reference path length, denoted by L_StartEnd, which is the length of the shortest path from START to END vertices, without passing through $V_{O B}$. It is assigned an infinite value if no such path exists. It is obvious that no path can pass through $V_{O W}$, the areas where wiring is not allowed. Since the reference path avoids buffer obstacle areas, buffers may be inserted anywhere along its route, hence ensuring that the end-to-end delay is the smallest possible. Thus any vertex found in a START to END path with a path length larger than the reference is redundant, because routing through this vertex produces a delay larger than that through the reference path. The above path lengths are computed by executing Dijkstra's algorithm. In the algorithm in Fig. 5, DIJKSTRA() function is invoked three times to obtain the required data used in graph pruning. The first call gives the distance (topological) of each vertex in $G$ to the sink vertex $E N D$, the second call gives the distances between the source vertex START to all vertices in $G$, and the third call gives another type of distance data, that is, distances from START to all other vertices, but this distances are through paths that exclude buffer obstacle vertices. From this distance, a reference distance $L \_S t a r t E n d$ is obtained.

\subsection{Look-ahead Scheme}

The runtime of S-RABILA is significantly improved by the application of a novel look-ahead scheme. The idea is to derive for each vertex, look-ahead weights which are non-dominated capacitance-delay pairs. These capacitance-delay pairs can be combined with any resistance-delay pair to obtain the end-toend delay. During path expansions, which are from source to sink, S-RABILA maintains resistance-delay pairs for all sub-paths. Availability of corresponding capacitance-delay pairs at each vertex permits the computation of an estimate of the end-to-end delay, and hence guides the path expansion. The challenge is how to compute these look-ahead weights (capacitance-delay pairs) for all vertices efficiently. The look-ahead scheme can be explained as follows. S-RABILA first transforms the original 2D graph into a 1D graph, and then computes the capacitance-delay pairs for all vertices in this $1 \mathrm{D}$ graph. A scheme is devised to assign look-ahead weights for all vertices in the original 2D graph, using the capacitance-delay pairs of the vertices in the $1 \mathrm{D}$ graph.

As an example, consider the sample problem given in Fig. 7, in which the dark area represents the area where wire is not allowed $\left(\mathrm{V}_{\mathrm{OW}}\right)$, and the grey area represents area where buffers are not allowed $\left(\mathrm{V}_{\mathrm{OB}}\right)$. Vertex-5 is the source and the sink is at vertex-4. We assume, without loss of generality, that only one wire size and one buffer type is available. We first determine the topological distance between the source vertex and the sink vertex, which is the length of the shortest path from source to sink that avoids the wire obstacle areas, but can pass through buffer obstacles. A corresponding 1D grid graph with length equal to the source-to-sink topological distance is created. This new graph has no obstacles whatsoever (neither wire nor buffer obstacles). For the example graph in Fig. 7, the source-to-sink topological distance is six. Fig. 8 shows the corresponding 1D grid graph with the grid length of six.

In this 1D grid graph, vertex-7 corresponds to the sink vertex in the original 2D graph. Vertex-6 corresponds to all the vertices in the original 2D graph that are one grid distance from the sink, while vertex-5 corresponds to vertices two grids away from the sink, and so on. The delay of the path from each vertex to the sink vertex is computed. Since the delay to be computed is with respect to the sink, the computation scheme using $(c, t)$ pairs is applied. The weight vectors (capacitance-delay pairs) for each vertex are computed using the conventional dynamic programming method, with only the non-dominated vectors stored for each vertex. As shown in the graph of Fig. 8, the weight vectors are listed under the respective vertex. In this example, we use the following parameter values: load capacitance at sink vertex 
is $0.022 \mathrm{pF}$, wire resistance is $37.5 \Omega$, wire capacitance is $0.1026 \mathrm{pF}$, buffer input capacitance is 0.022 $\mathrm{pF}$, buffer output resistance is $104.2 \Omega$, and intrinsic buffer delay is $20 \mathrm{ps}$. We also assume that the source has an output resistance of $104.2 \Omega$.

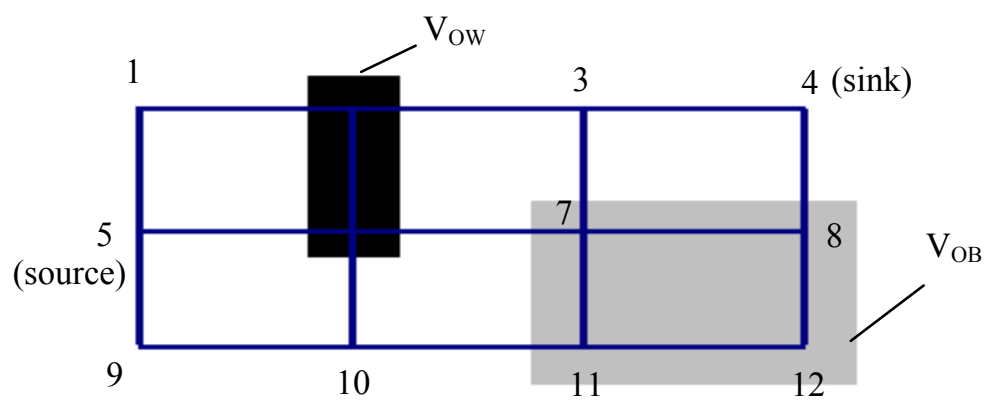

Fig. 7. Sample problem to illustrate look-ahead concept.

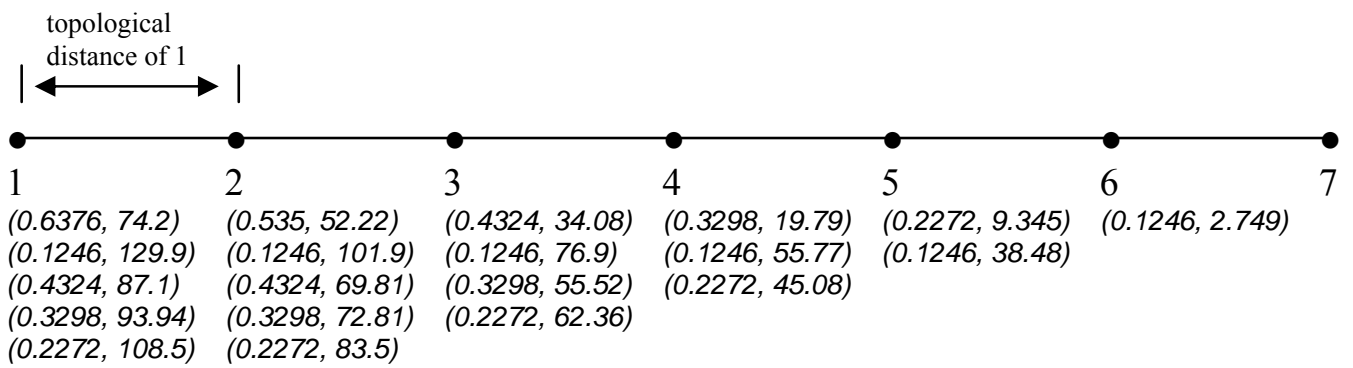

Fig. 8. 1-dimensional graph with look-ahead weight vectors, $(c, t)$ (unit for $c$ is $\mathrm{pF}$ while unit for $t$ is ps.)

Note again that the 1D graph has no obstacles (i.e. neither wire nor buffer obstacles). It is clear then, that the weight vectors stored at each vertex in the 1D grid graph provide the absolute lower bound of the delay from a vertex to the sink, since buffer can be inserted anywhere as necessary along the path. For example, consider vertex-1 in the 1D graph (Fig. 8), which corresponds to all vertices six grids away from the sink in the 2D graph (Fig. 7). Then, the look-ahead weights at vertex-1 in the 1D graph are nondominated, absolute lower bound weight vectors from the sink to a vertex six-grid distance away in the original 2D grid graph. Hence these vectors can be viewed as look-ahead weights. The look-ahead weights at vertex $u$ in the $2 \mathrm{D}$ graph are denoted by WeightLA[u] in our algorithm, and the number of these weights associated with a vertex $u$ is denoted by LACount $[u]$.

Note that the look-ahead 1D grid graph is based on the source-to-sink topological distance. Consequently, vertices further away from the sink will not have any look-ahead vectors. For example, the distance of vertex-1 to the sink in the original $2 \mathrm{D}$ graph is 7 . This distance exceeds the topological source-to-sink distance, which is only six. Look-ahead vectors are not calculated for these "far-away" vertices. A special value, WeightMax, is assigned as the look-ahead weight for these far-away vertices. WeightMax is the minimum end-to-end delay for the 1D graph, after taking into account the source resistance, and is given by: WeightMax $=\min \left(R_{\text {Source }}{ }^{*} c+t, \forall(c, t)\right.$ weights at vertex 1$)$, where $R_{\text {Source }}$ is the source resistance. For the example problem in Fig. 7, where $R_{\text {Source }}=104.2 \Omega$, the minimum delay is $128.3 \mathrm{ps}$, which is obtained using capacitance-delay pair $(0.3298,93.94)$. This constant is now used as the look-ahead delay for all vertices in the original 2D grid graph with topological distance from the sink greater than six. Now, all vertices in the 2D graph will have its own set of look-ahead vectors. Fig. 9, which is a redraw of Fig. 7, illustrates the association between vertices in the 1D graph and the original $2 \mathrm{D}$ graph.

For example, vertex-11 is three grids away from the sink; therefore it is associated with vertex-4 in the corresponding 1D grid graph. Hence, the look-ahead weights of vertex-11, WeightLA[11] $=\{(0.3298$, 
19.79), $(0.1246,55.77),(0.2272,45.08)\}$. These three weight vectors represent non-dominated $(c, t)$ pairs, one of which is due to path expansion with buffer insertion at vertex-5 in $1 \mathrm{D}$ grid graph. When the same look-ahead weight vectors are passed on to vertex-11 in the original 2D graph, similarly, one of the weight vectors assigned to vertex-11 is associated with buffer insertion at vertex-7. Since vertex-7 actually cannot have buffer insertion, one of the look-ahead weight vectors for vertex-11 is not valid. Since it is too troublesome to tract which vertex in the 1D graph that may correspond to vertices in the 2D graph that have restrictions on buffer locations, we just assume that buffer can be inserted anywhere and the non-valid look-ahead weight vectors are also assigned to the corresponding $2 \mathrm{D}$ vertices. The algorithm will handle the non-valid weight vectors such that optimal delay can still be computed.

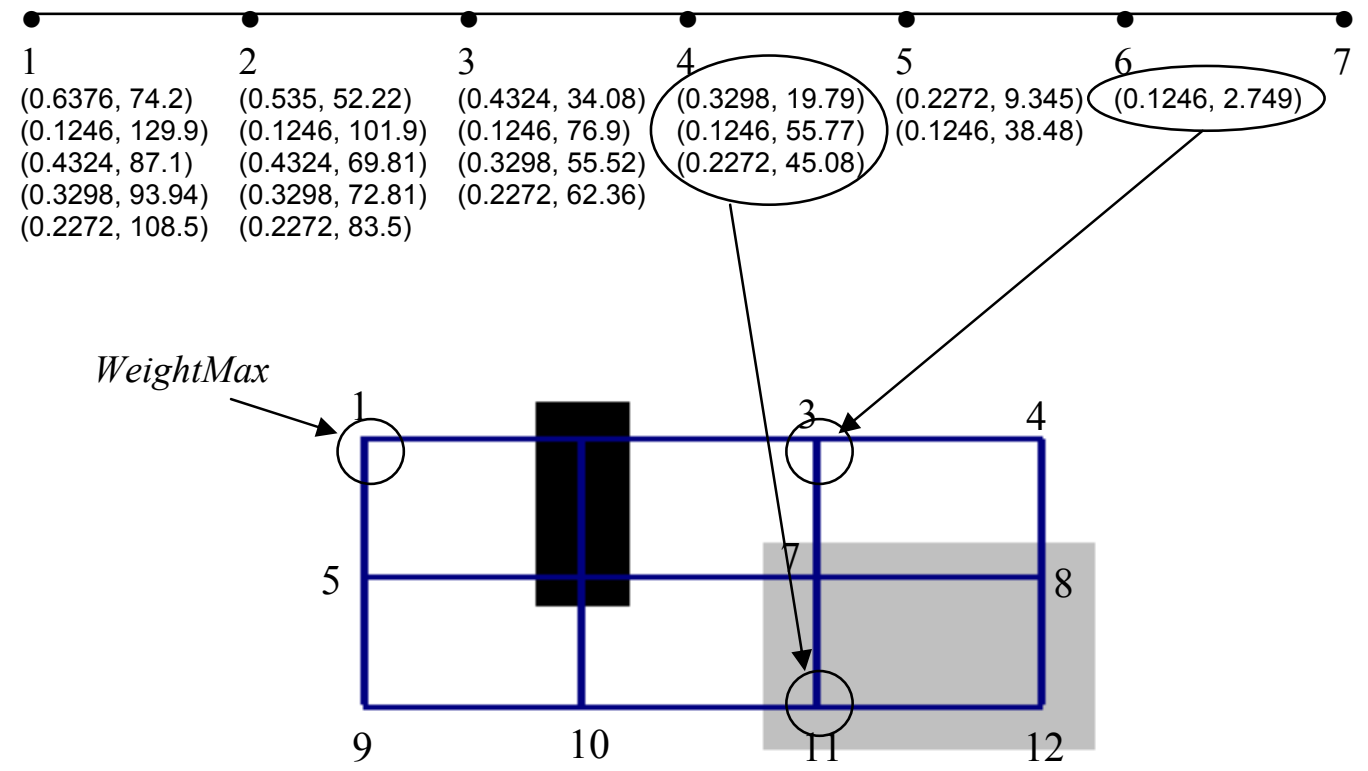

Fig. 9. Association of look-ahead weight vectors to input grid graph (source is vertex-5 and vertex-4 is the sink).

The above discussion suggests that we can utilize the look-ahead weights as an estimate of actual $(c, t)$ pairs for the vertices in the $2 \mathrm{D}$ graph. Consequently, when we have computed the $(r, t)$ value for a candidate, and knowing the $(c, t)$ pairs for the candidate's vertex, we can then calculate the (predicted) end-to-end delay, EndDelay, by using Equation 5. If EndDelay is greater than the actual known minimum source-to-sink delay, then this candidate is considered dominated, and therefore is removed. In this way, the number of candidates at the vertices can be substantially reduced, thus speeding up the process of constructing the routing path significantly.

\subsection{EXPERIMENTAL RESULTS}

Here we report three benchmarking experiments conducted to measure the performance of S-RABILA. The tests are conducted on a PC with a $3 \mathrm{GHz}$ Pentium D processor and 504 MB RAM.

In the first experiment, we benchmark S-RABILA against DP algorithm [6], which has been introduced in Section 2. The performance metrics used in the comparison of the two algorithms are algorithm execution runtime $(T)$, and the total number of sub-path candidates that are created $(N)$. The parameters used here represent typical interconnect wires used in a $65 \mathrm{~nm}$ fabrication process. We select six wires with wire capacitance in the range $16.7-36.7 \mathrm{fF}$, and $1.4-44.9 \Omega$ for wire resistance. Similarly, six buffers are chosen. The buffer parameters are as follows: $20 \mathrm{fF}<c_{b}<32.2 \mathrm{fF}, 118.1 \Omega<r_{b}<200 \Omega, 10$ ps $<d_{b}<75.9$ ps. Two randomly generated test graphs are used in this performance test: Test graph 1 and Test graph 2. Test graph 1 is a small-size graph with a $22 \times 17$ grid, $44.4 \%$ buffer obstacle area and $26.7 \%$ wire obstacle area. Test graph 2 is a medium-size graph with an $80 \times 40$ grid, $26 \%$ buffer obstacle area and $5.3 \%$ wire obstacle area. The algorithm is run for six cases, which are differentiated by the size of 
wire/buffer library (we set the wire library size = buffer library size). The results are shown in Fig. 10(a) and (b) for Test graph 1. Fig. 11(a) and (b) show the results of executing the algorithms on Test graph 2. The results show that S-RABILA executes significantly faster than DP. The runtime of S-RABILA is linear to the library size, whereas it is exponential for DP. This clearly indicates that the look-ahead scheme in S-RABILA contributes significantly to the computational effectiveness of the algorithm, as SRABILA is similar in execution as DP when it is without the look-ahead feature.

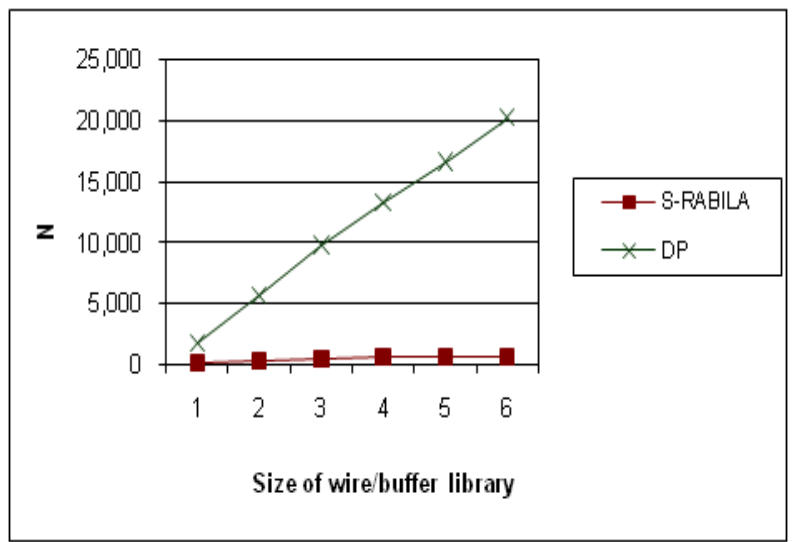

(a)

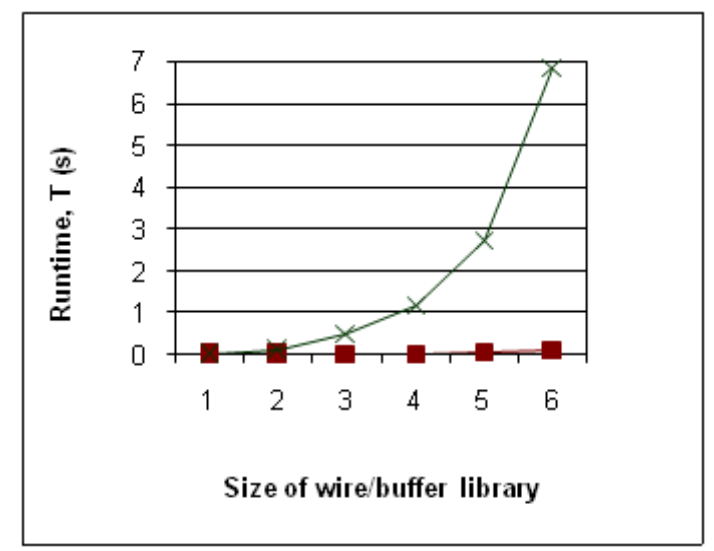

(b)

Fig. 10. Small-size Graph 1. (a) Plot of $N$ versus library size. (b) Plot of runtime $(T)$ versus library size.

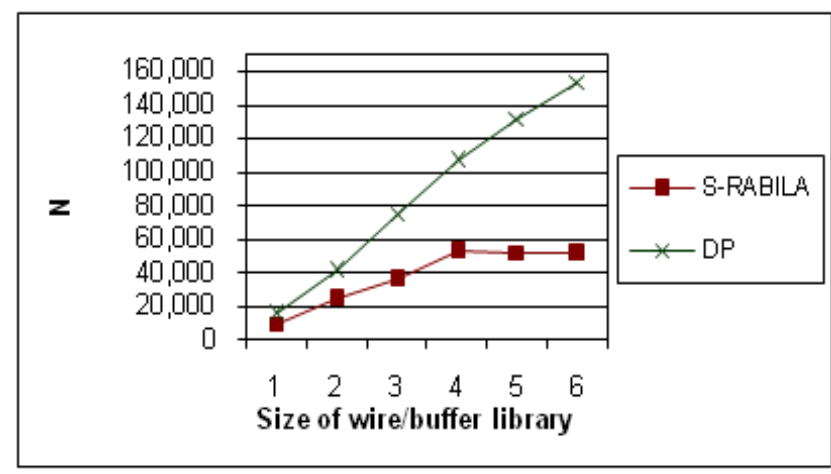

(a)

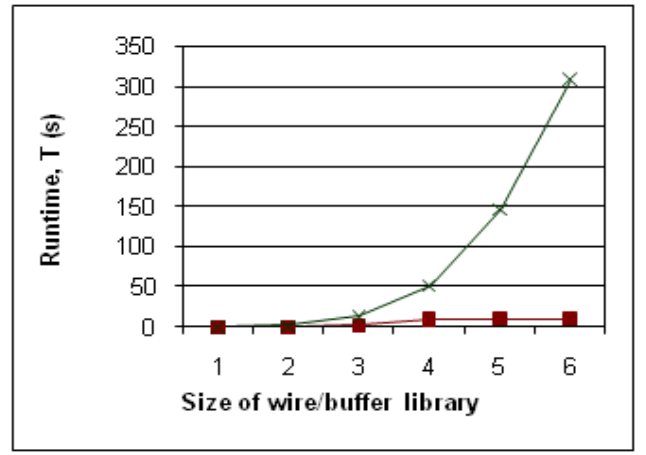

(b)

Fig. 11 Medium-size Graph 2. (a) Plot of $N$ versus library size. (b) Plot of runtime $(T)$ versus library size.

In the second experiment, we benchmark S-RABILA against the SP (Shortest Path algorithm) by [3], which has been described in Section 2. In [3], the authors perform a runtime comparison between their SP algorithm and the DP algorithm. They used eight different graph sizes in that comparison, with three buffers and five different wires. The value of wire capacitances that were chosen was in the range $22.3-$ $102.6 \mathrm{fF}$, and $6.9-37.5 \Omega$ for wire resistance. The buffer parameters are as follows: $22 \mathrm{fF}<\mathrm{c}_{\mathrm{b}}<158.4$ $\mathrm{fF}, 104.2 \Omega<\mathrm{r}_{\mathrm{b}}<1064.1 \Omega, 20 \mathrm{ps}<\mathrm{d}_{\mathrm{b}}<40 \mathrm{ps}$. The source resistance and load capacitance was $104.2 \Omega$ and $158.4 \mathrm{fF}$ respectively. Lai and Wong did provide the delay that they obtained for each graph. Based on all these information, a graph that should be more or less equal to what they had can be approximated. We created eight graphs that are similar to the graphs used by Lai and Wong to be used as the test graphs in this performance test on algorithm runtime $\mathrm{T}$. The wire and buffer parameters that are used in the tests are provided in Tables 1 and 2 . 
Table 1: Wire library from [3]

\begin{tabular}{|l|l|l|l|}
\hline Name & $\begin{array}{l}\text { Length } \\
(\mu \mathrm{m})\end{array}$ & $\begin{array}{l}\mathrm{r}_{\mathrm{w}} \\
(\Omega)\end{array}$ & $\begin{array}{l}\mathrm{C}_{\mathrm{w}} \\
(\mathrm{fF})\end{array}$ \\
\hline Wire1 & 500 & 37.5 & 22.2 \\
\hline Wire2 & 500 & 30 & 42 \\
\hline Wire3 & 500 & 22 & 62 \\
\hline Wire4 & 500 & 15 & 83 \\
\hline Wire5 & 500 & 6.9 & 102.6 \\
\hline
\end{tabular}

Table 2: Buffer library from [3]

\begin{tabular}{|l|c|c|c|}
\hline Name & $\begin{array}{c}\text { Intrinsic } \\
\text { delay } \mathrm{d}_{\mathrm{b}} \\
(\mathrm{ps})\end{array}$ & $\begin{array}{c}\text { Output } \\
\text { resistance } \mathrm{r}_{\mathrm{b}} \\
(\Omega)\end{array}$ & $\begin{array}{c}\text { Input } \\
\text { cap } \mathrm{c}_{\mathrm{b}} \\
(\mathrm{fF})\end{array}$ \\
\hline Buf1 & 40 & 1064.1 & 22 \\
\hline Buf2 & 30 & 584 & 90 \\
\hline Buf3 & 20 & 104.2 & 158.4 \\
\hline
\end{tabular}

The results are summarized in Table 3. It can be concluded that S-RABILA is faster than SP algorithm. For example, in the case of the $22 \times 32$ graph, we can deduce that S-RABILA is $21.5(66.7 / 3.1=21.5)$ times faster than SP. The authors exclude the overhead time for determining the weights for the edges in their BP graph in the SP runtime results. In other words, the SP runtime measure only the time taken for their Dijkstra's algorithm to solve the new BP graph after edge costs had been already assigned. The performance of SP algorithm probably is slightly worse if the overhead time is taken into account.

Table3: Runtime comparison between DP, SP, and S-RABILA algorithms

\begin{tabular}{|c|c|c|c|c|c|c|}
\hline \multirow{2}{*}{ Graph size } & \multicolumn{2}{|c|}{ Runtime (s) } & \multicolumn{2}{c|}{ Runtime (s) } & \multicolumn{2}{c|}{$\begin{array}{c}\text { Runtime improvement } \\
\text { over DP }\end{array}$} \\
\cline { 2 - 7 } & DP & SP & DP & S-RABILA & SP & S-RABILA \\
\hline $20 \times 24$ & 148.1 & 5.4 & 11.17 & 0.2637 & $27.4 \times$ & $42.4 \times$ \\
\hline $28 \times 22$ & 197.1 & 93.2 & 23.01 & 2.148 & $2.1 \times$ & $10.7 \times$ \\
\hline $20 \times 30$ & 231.2 & 20.4 & 29.45 & 0.7203 & $11.3 \times$ & $40.9 \times$ \\
\hline $22 \times 32$ & 303.5 & 97 & 72.55 & 1.088 & $3.1 \times$ & $66.7 \times$ \\
\hline $28 \times 28$ & 258.2 & 63.8 & 28.21 & 0.8903 & $4.0 \times$ & $31.7 \times$ \\
\hline $28 \times 24$ & 269.5 & 25.3 & 51.04 & 0.6726 & $10.7 \times$ & $75.9 \times$ \\
\hline $24 \times 28$ & 213.6 & 11.1 & 18.88 & 0.522 & $19.2 \times$ & $36.2 \times$ \\
\hline $24 \times 20$ & 124.4 & 12.6 & 11.37 & 0.5777 & $9.9 \times$ & $19.7 \times$ \\
\hline
\end{tabular}

In the third experiment, S-RABILA is tested on a large graph, Test graph 3, as shown in Fig. 12. Its characteristics are summarized in Table 4 . The same wire and buffer parameters as in the first experiment are used. The algorithm is run for six cases, which are differentiated by the size of buffer library. In all six cases, only Wirel is used. The results of executing S-RABILA on Test graph 3 are shown in Fig. 13(a) and (b). The runtime of S-RABILA in this test is again not exponential to the buffer library size. It seems to be linear for the first three cases, after which the runtime performance even improves slightly. Unfortunately, however, this linearity apparently not applicable anymore when wiresizing is to be performed. When the wire library is increased, the runtime is simply too long. In conclusion, we can assert that for most practical cases, the runtime for S-RABILA is linear to problem size.

Table 4: Characteristics of Test graph 3 (large graph)

\begin{tabular}{|c|c|c|c|c|c|c|}
\hline Size & $\begin{array}{c}\text { Equivalent } \\
\text { layout area }\end{array}$ & $\begin{array}{c}\text { Source } \\
\text { vertex }\end{array}$ & $\begin{array}{c}\text { Sink } \\
\text { vertex }\end{array}$ & $\begin{array}{c}\text { Buffer } \\
\text { obstacle }\end{array}$ & $\begin{array}{c}\text { Wire } \\
\text { obstacle }\end{array}$ & $\begin{array}{c}\text { Effective wire } \\
\text { obstacle }\end{array}$ \\
\hline $300 \times 200$ & $30 \times 20 \mathrm{~mm}^{2}$ & 18940 & 41320 & $30 \%$ & $30 \%$ & $59.2 \%$ \\
\hline
\end{tabular}




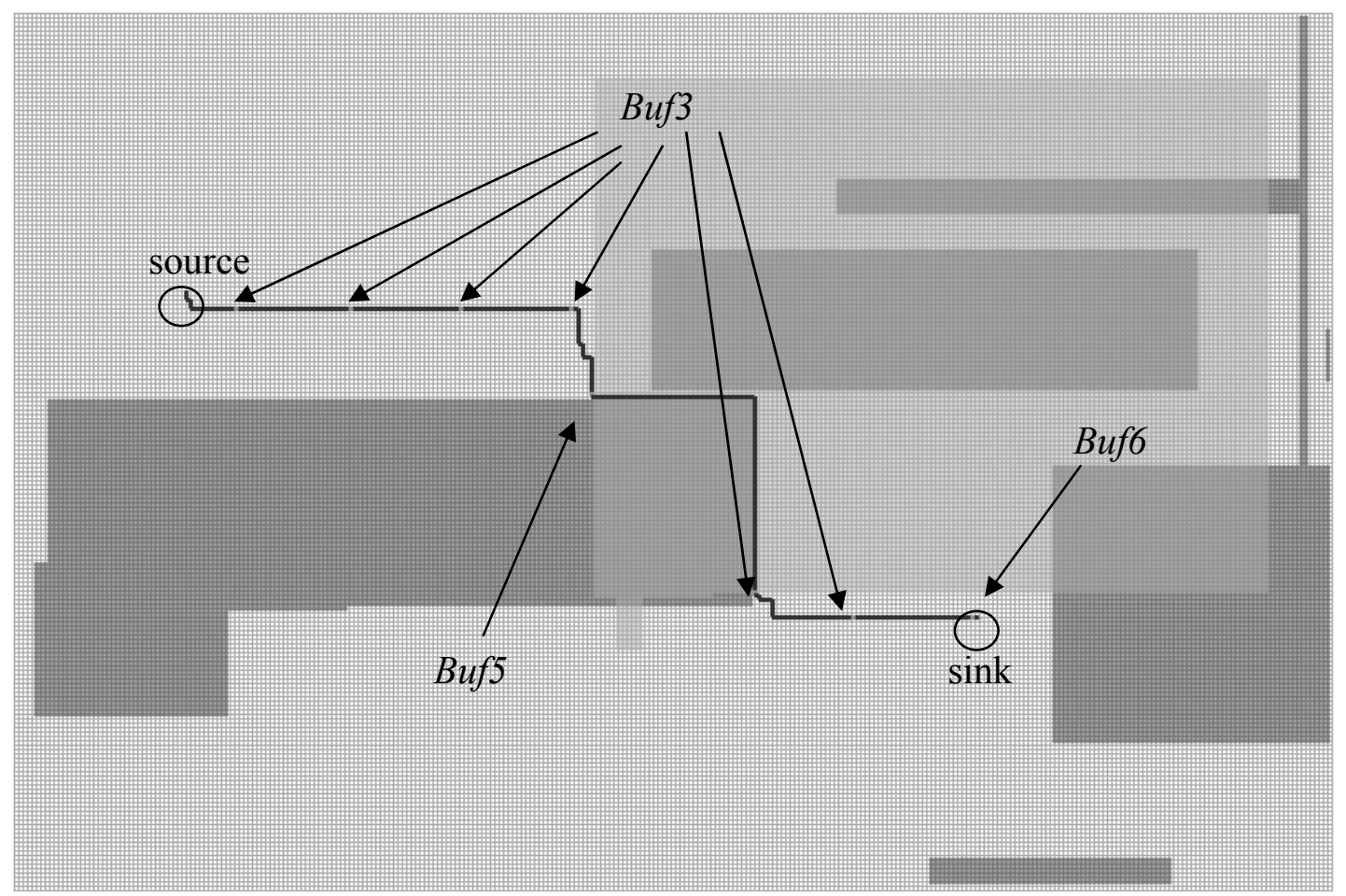

Fig. 12 Large-size Graph (test graph 3)

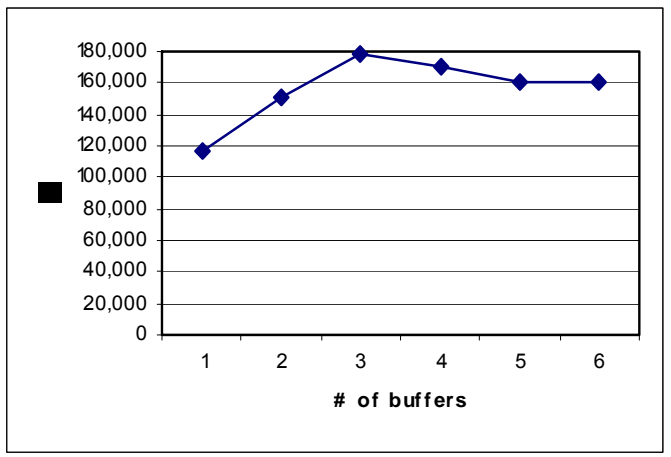

(a)

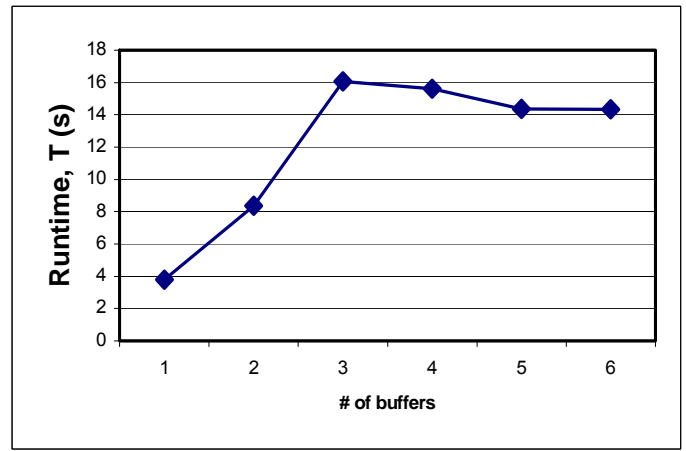

(b)

Fig. 13 Large-size Graph 3. (a) Plot of $N$ versus library size. (b) Plot of runtime ( $T$ ) versus library size.

\section{CONCLUSION}

This paper describes a new algorithm for optimal interconnect delay for two-terminal nets in VLSI layout routing design. This algorithm, named S-RABILA, inserts buffers while considering wire and buffer obstacles and simultaneously constructs interconnect routing with wire sizing. The search mechanism in the algorithm is based on the van Ginneken dynamic programming approach. The key contribution of the work is a novel look-ahead scheme, which has been shown to improve significantly the computational speed of the search in S-RABILA. In most of the performance tests, the runtime of S-RABILA is an order of magnitude faster than DP. Also, when compared to SP routing algorithm, it is at least 1.5 times faster. This speed improvement clearly indicates the computational effectiveness of the look-ahead scheme. Other tests performed have also shown that S-RABILA can handle large graphs with acceptable runtimes. Thus, it has been shown that S-RABILA is accurate, fast, scalable with problem size, and can handle large routing graphs. 


\section{Acknowledgement}

This research is supported in part by the funding from Intel Technology Sdn Bhd, Penang, UTM Vote No. 73316.

\section{REFERENCES}

[1] L. P. P. P. van Ginneken, "Buffer placement in distributed RC-tree networks for minimal Elmore delay", in Proc. Int. Symp. Circuits and Systems, 1990, pp. 865-868.

[2] J. Cong et al., "Performance optimization of VLSI interconnect layout", INTEGRATION, the VLSI Journal, vol. 21, pp. 1-94, 1996.

[3] M. Lai and D. F. Wong, "Maze routing with buffer insertion and wiresizing", IEEE Trans. Computer-Aided Design of Integrated Circuits and Systems, vol. 21, pp. 1205-1209, Oct. 2002.

[4] Z. Li and W. Shi, "An O $\left(\mathrm{bn}^{2}\right)$ time algorithm for optimal buffer insertion with b buffer types", IEEE Trans. Computer-Aided Design of Integrated Circuits and Systems, vol. 25, pp. 484-489, 2006.

[5] J. Lillis, C.-K. Cheng, and T.-T. Y. Lin, "Optimal wire sizing and buffer insertion for low power and a generalized delay model", IEEE Journal of Solid-State Circuits, vol. 31, pp. 437-447, 1995.

[6] H. Zhou et al., "Simultaneous routing and buffer insertion with restrictions on buffer locations", IEEE Trans. Computer-Aided Design of Integrated Circuits and Systems, vol. 19, pp. 819-824, July 2000.

[7] L-D. Huang et al., "Maze routing with buffer insertion under transition time constraints", IEEE Trans. Computer-Aided Design of Integrated Circuits and Systems, vol. 22, pp. 91-95, 2003.

[8] P. Van Mieghem and F. A. Kuipers, "Concepts of exact QoS routing algorithms", IEEE/ACM Trans. Networking, vol. 12, no. 5, pp. 851-864, Oct. 2004.

[9] J. M. Rabaey, A. Chandrakasan, and B. Nikolić, Digital Integrated Circuits: A Design Perspective. $2^{\text {nd }}$ ed., Upper Saddle River, NJ, Pearson Education, Inc., 2003.

[10] T. H. Cormen, C. E. Leiserson, and R. L. Rivest, Introduction to Algorithms, Cambridge, MA, MIT Press, 1990.

[11] P. E. Hart, N. J. Nilsson, and B. Raphael, "A formal basis for the heuristic determination of minimum cost paths", IEEE Trans. Systems Science and Cybernetics, vol. 4, no. 2, pp. 100-107, 1968. 